\title{
SALUD ORAL EN ADULTOS JÓVENES PERUANOS
}

\section{ORAL HEALTH IN YOUNG PERUVIAN ADULTS}

\author{
Aquino-Canchari Christian R. ${ }^{1 *}$ Huamán-Castillón Katia M. $^{2}$ \\ ${ }^{1}$ Especialidad en Salud Pública Estomatológica y Salud Colectiva, Maestría en Estomatología (Universidad Peruana \\ Cayetano Heredia, Perú). Sociedad Científica Médico Estudiantil Peruana (SOCIMEP). Perú \\ ${ }^{2}$ Maestría en Gestión de Salud (Universidad Continental, Perú). Cirujano Dentista en la Región de Sanidad PNP \\ Huancavelica. Perú \\ * christian.aquino.canchari@gmail.com
}

\begin{abstract}
Resumen
OBJETIVO: Determinar la prevalencia, experiencia, significancia de caries dental y la condición de higiene oral en alumnos de la Escuela de Educación Superior Técnico Profesional PNP, en el año 2017. MATERIALES Y MÉTODOS: Estudio observacional, descriptivo, transversal. La muestra estuvo conformada por 174 adultos jóvenes seleccionados aleatoriamente, cumpliendo criterios de inclusión y exclusión y siguiendo las normas éticas en investigación. Los datos se analizaron en el programa SPSS 23 mediante tablas de distribución de frecuencias. RESULTADOS: La prevalencia de caries dental fue de $98.50 \%$ (CPO-D = 5,76; SIC=9,76 y una condición de higiene oral con un predominio de los niveles malo y regular. CONCLUSIONES: El estudio evidenció la necesidad de desarrollar programas preventivos promocionales coherentes, dinámicos de salud bucal en adultos jóvenes de la población de estudiantes estudiados .
\end{abstract}

Palabras clave: salud bucal, caries dental, adulto, Perú.

\begin{abstract}
AIM: To determine the prevalence, experience, significance of dental caries and the condition of oral hygiene in students of the PNP Professional Technical Higher Education School, in 2017. MATERIALS AND METHODS: Observational, descriptive, cross-sectional study. The sample consisted of 174 randomly selected young adults, meeting inclusion and exclusion criteria and following the ethical norms in research. The data was analyzed in the SPSS 23 program by means of frequency distribution tables. RESULTS: The prevalence of dental caries was 98.50\% (CPO-D = 5.76, SIC $=9.76$, and oral hygiene condition of bad and regular predominance.) CONCLUSION: The study evidenced the need to develop coherent promotional preventive programs. , dynamic oral health in young adults.
\end{abstract}

Key words: oral health, dental caries, adult, Peru.

\section{INTRODUCCIÓN}

La etapa de vida de adulto joven se caracteriza porque comienza a asumir responsabilidades de índole social y familiar, iniciando el desarrollo de su proyecto de vida. ${ }^{1}$ Las enfermedades bucodentales, infecciones de las vías respiratorias superiores, infecciones de transmisión sexual son los más prevalentes según lo reportado por el Ministerio de Salud del Perú (MINSA), ${ }^{2}$ es así que las intervenciones en prevención de la enfermedades y promoción de hábitos saludables son importantes ya que las decisiones asumidas en esta etapa de vida tendrán consecuencias y efectos favorables o desfavorables en el desarrollo de su salud por el resto de su vida. ${ }^{3,4}$

La Organización Mundial de la Salud (OMS) establece a la salud bucodental, como factor primordial para tener una adecuada salud general y buena calidad de vida, la Organización
Mundial de la Salud la define como la "ausencia de dolor orofacial, cáncer de boca o de garganta, infecciones bucales, periodontopatías, caries, pérdida de dientes, traumatismos y otras enfermedades y trastornos que limitan en la persona afectada la capacidad de hablar, morder, masticar y sonreír, al tiempo que generan un impacto en su bienestar psicosocial". 5

La caries dental es un problema sanitario mundial afectando tanto a los países desarrollados como a los que están en vías de desarrollo. ${ }^{6,7}$ La OMS define la caries dental como un proceso localizado de origen multifactorial (huésped, dieta, microorganismos, tiempo). ${ }^{8}$

La región de las Américas es donde se encuentran los problemas más álgidos en cuanto a la salud bucal, siendo la más prevalente e incidente la caries dental a nivel mundial, el Perú no escapa a esta realidad, según lo reportado por el MINSA 
en el año 2005, evidencia una prevalencia de caries dental de $90 \%$ en escolares. Siendo la prevalencia en población urbana $(90,6 \%$ y en población rural $(88,7 \%)$ referente al índice ceod/ CPO-D a nivel nacional fue de 5,84. ., $^{9}$

Es así que se ha establecido el plan nacional de salud $2007-$ 2020 estableciendo como indicador sanitario la disminución de enfermedades bucodentales, reducir el índice de CPODceod, atención bucal basada en la Atención Primaria de Salud Renovada (APS-R), esto se traduce en una disminución del gasto en salud generado por la atención recuperativa. ${ }^{11}$

El objetivo del estudio fue determinar la significancia, experiencia de caries dental y el nivel de higiene oral en adultos jóvenes de la Escuela Técnica Superior PNP Huancavelica, departamento de Huancavelica, Perú, 2017.

\section{MATERIALES Y MÉTODOS}

Diseño de estudio tipo observacional, descriptivo de corte transversal. La muestra estuvo conformada por 174 adultos jóvenes de sexo masculino de 18 a 24 años de edad pertenecientes a la Escuela Técnica Superior PNP, Huancavelica, durante el 2017.

Para establecer la prevalencia y experiencia de la caries dental se cuantificó con el índice CPOD de la OMS, ${ }^{12}$ para el grado de severidad se utilizó el índice de Grimep, ${ }^{13}$ la condición de higiene bucal, se midió en base al índice de Greene y Vermillion modificado (IHO-S). ${ }^{14}$ Estos instrumentos fueron validados en un estudio piloto con un grupo de adultos jóvenes con características similares, haciéndose los ajustes necesarios, para la recolección de estos datos se utilizó una ficha epidemiológica dental conteniendo un odontograma.

El criterio de inclusión fue: consentimiento informado, los criterios de exclusión fueron: presencia de alguna enfermedad sistémica. Para la recolección de datos se utilizaron criterios de la OMS. ${ }^{15}$ Utilizando luz natural y equipos de diagnóstico para la detección de caries dental, mediante la técnica de observación directa.

Los evaluadores fueron sometidos a calibración y pruebas de concordancia, los resultados fueron sometidos al índice de Kappa de Cohen para establecer la concordancia entre los observadores, obteniendo el valor 0,94.

Los datos fueron analizados en el programa Microsoft Excel y posteriormente se realizó la cuantificación estadística mediante el paquete estadístico SPSS 23, para el análisis descriptivo se obtuvo porcentajes y medidas de frecuencia, puntajes promedios y desviaciones estándar de las variables.

\section{RESULTADOS}

De los 174 adultos jóvenes evaluados; el promedio de edad de la población fue de 20,71 años de edad y el rango de edad varió de 18 a 24 años de edad. (Tabla 1) Se encontró una prevalencia de caries dental de $98,50 \%$ la experiencia de caries dental poblacional fue alto $(5,76)$ según los criterios de la OMS y un SIC de (Tabla 2)

Tabla 1.- Muestra según edad.

\begin{tabular}{ccc}
\hline Edad & $\mathrm{n}$ & $\%$ \\
\hline 18 & 24 & 13,79 \\
19 & 29 & 16,66 \\
20 & 27 & 15,51 \\
21 & 38 & 21,83 \\
22 & 20 & 11,49 \\
23 & 21 & 12,06 \\
24 & 15 & 8,66 \\
Total & 174 & 100 \\
\hline
\end{tabular}

Tabla.-2: Prevalencia de Caries Dental

\begin{tabular}{lcc}
\hline Indicador & Valor & \\
\hline Masculino (n=174) & & \\
Prevalencia n (\%) & 172 & $(98,50 \%)$ \\
Diente Cariado Me ( D.E) & 4,11 & $(3,52)$ \\
Diente Extraído Me ( D.E) & 0.95 & $(1,29)$ \\
Diente Obturado (D.E.) & 7,28 & $(5,14)$ \\
SIC & 9,76 & \\
\hline
\end{tabular}

Me. Media

Tabla 3.-Condicion de Higiene Oral

\begin{tabular}{ccccccc}
\hline & \multicolumn{2}{c}{ Bueno } & \multicolumn{2}{c}{ Regular } & \multicolumn{2}{c}{ Malo } \\
Edad & $\mathbf{n}$ & $\boldsymbol{\%}$ & $\mathbf{n}$ & $\boldsymbol{\%}$ & $\mathbf{n}$ & $\%$ \\
\hline 18 & 5 & 20,80 & 7 & 29,20 & 12 & 50,00 \\
19 & 5 & 17,20 & 13 & 44,80 & 11 & 37,90 \\
20 & 6 & 22,20 & 10 & 37,01 & 1 & 40,69 \\
21 & 5 & 13,20 & 17 & 44,70 & 16 & 42,10 \\
22 & 5 & 25,00 & 6 & 30,00 & 9 & 45,00 \\
23 & 7 & 33,30 & 9 & 42,90 & 4 & 23,80 \\
24 & 3 & 20,00 & 7 & 46,70 & 5 & 33,30 \\
Total & $\mathbf{3 6}$ & $\mathbf{2 0 , 7 0}$ & $\mathbf{6 9}$ & $\mathbf{3 9 , 7 0}$ & $\mathbf{6 9}$ & $\mathbf{3 9 , 7 0}$ \\
\hline
\end{tabular}

\section{DISCUSIÓN}

Los resultados evidencian la problemática en salud bucal y concuerda con el panorama de la región de las Américas. ${ }^{16}$ La prevalencia de caries dental encontrada en este estudio fue de $98.5 \%$, siendo mayor a lo reportado en 2017 por Kramer A et al. (47\%), ${ }^{17}$ Drachev SN, Brenn T, Trovik TA $(96 \%),{ }^{18}$ Gonzáles A et al en $2009(90 \%),{ }^{19}$ a pesar de las mejoras en la atención bucodental en el sistema sanitario peruano aún quedan brechas en cuanto al acceso, accesibilidad a los servicios de salud, recurso humano capacitado en APS-R. ${ }^{20}$ La Organización Panamericana de Salud (OPS) establece el Índice CPOD en relación a la severidad siendo lo valores: muy bajo $(0,0-1,1)$, bajo $(1,2-2,6)$, moderado $(2,7-$ $4,4)$ y alto $(4,5-6,5)$ y muy alto $\left(6,6\right.$ a más). ${ }^{21}$ En relación al CPOD encontrado, este fue mayor a lo reportado por Spencer 
AJ, Liu P, Do LG en 2017 (5,57), ${ }^{22}$ García-Cortés JO et al. en 2014 (4,24), ${ }^{23}$ Arrieta K, Gonzáles F, Díaz S en 2012 $(4,2),{ }^{24}$ este panorama reafirma la necesidad de desarrollar programas preventivos coherentes, dinámicos, eficaces, desde edades tempranas. Referente a la condición e higiene oral predominante fue la regular y mala siendo diferente a lo reportado Gómez N, Morales M donde la condición buena fue la más predominante $(81,4 \%)^{25}$ y similar a lo reportado por Doncel $\mathrm{C}$ et al. en 2011 donde la condición regular fue la más predominante $(82 \%),{ }^{26}$ debemos remarcar que una adecuada higiene dental contribuye a la disminución de periodontopatías.

\section{CONCLUSIÓN}

La prevalencia, experiencia, significancia de caries dental los adultos jóvenes fue 98,5\%; 5,76; 9,76; respectivamente, referente a la condición de higiene oral la mayoría presen- 1 to la condición mala y regular. Estos resultados muestran la urgencia de desarrollar políticas sanitarias bucales basadas en 15 un enfoque preventivo promocional en este grupo etareo.

CONFLICTO DE INTERESES Los autores no tienen ningún conflicto de interés.

\section{Referencias}

1 Petrera M. Algunas reflexiones en torno a las cuentas nacionales de salud del Perú. Rev Perú Med Exp Salud Pública. 2009; 26(2): 248-50.

2 Blanco M, Padrón J, Pachón L, Sánchez T, Medina R. Educación para la salud integral del adolescente a través de promotores pares. Rev. Med. Electrón 2011; 33(3): 349-359.

3 Giraldo A, Toro M, Macías M, Valencia C, Palacio S. La promoción de la salud como estrategia para el fomento de estilos de vida saludables. Hacia Promoc. Salud 2010; 15(1): 128-143.

4 Organización Panamericana de la Salud. Carta de Ottawa para la Promoción de la Salud. Ottawa: OPS; 1986.

5 Organización Mundial de la Salud. Salud Bucodental. Centro de prensa. Nota informativa $\mathrm{N}^{\mathrm{a}}$ 318. Febrero 2007[acceso 16 de Enero del 2018]. Disponible en: http://www.who.int/ mediacentre/factsheets/fs318/es/

6 Medina-Solís C, Maupomé G, Pelcastre-Villafuerte B, Avila-Burgos L, Vallejos-Sánchez A, Casanova-Rosado A. Desigualdades socioeconómicas en salud bucal: caries dental en niños de seis a 12 años de edad. Rev. Invest. Clín. 2006; 58(4): 296-304.

7 Abadía Barrero César Ernesto. Pobreza e Desigualdes Sociais: Um debate obrigatorio em saude oral. Acta Bioéth. 2006; 12(1): 9-22.

8 Portilla J, Pinzón ME, Huerta ER, Obregón A. Conceptos actuales e investigaciones futuras en el tratamiento de la caries dental y control de la placa bacteriana. Rev. Odont. Mex 2010; 14(4): 218-225.

9 Ministerio de Salud. Prevalencia nacional de caries dental, fluorosis del esmalte y urgencia de tratamiento en escolares de 6 a 8, 10, 12 y 15 años. Lima, Perú: MINSA; 2002.

10 Ministerio de Salud. Documento Técnico Plan Nacional de Salud Bucal Sonríe Siempre Perú. 2001- 2002. Lima, Perú: MINSA; 2005.

1 Cam LE. Fluorización de la sal en el Perú. 2006 [acceso 16 de enero del 2018] Disponible en: http://odontorural.wordpress.com/2006/08/24/ fluorización-de-la-sal-en-el-Peru/al

12 World Health Organization (1987): Oral Health Surveys. Basic Methods. 3rd. Geneve, Suiza, WHO.

13 Andrade M, De la Cruz D. Indicadores de prevalencia y de predicción de riesgo de caries dental. Vertientes Revista Especializada en Ciencias de la Salud 2014, 17(1):61-72.

4 Greene J, Vermillion J. The simplified oral hygiene índex. J Am Dent Assoc. 1964 68(1): 7-13.

5 Pitts NB. Clinical diagnosis of dental caries: a European perspective. J Dent Educ 2001; 65(1):972-8.

16 Nithila A, Bourgeois D, Barmes DE, Murtomaa H. Banco Mundial de Datos sobre Salud Bucodental de la OMS, 19861996: panorámica de las encuestas de salud bucodental a los 12 años de edad. Rev Panam Salud Pública. 1998; 4(6):4115.

17 Kramer A, Petzold M, Hakeberg M, Östberg AL. Multiple Socioeconomic Factors and Dental Caries in Swedish Children and Adolescents. Caries Res. 2017 14; 52(1-2):42-50.

18 Drachev SN, Brenn T, Trovik TA. Dental caries experience and determinants in young adults of the Northern State Medical University, Arkhangelsk, North-West Russia: a crosssectional study. BMC Oral Health. 2017; 17(1):136.

19 González A, Martínez T, Alfonzo N, Rodríguez J, Morales A. Dental caries and risk factors present in young adults. Rev Cubana Estomatol. 2009; 46(3):30-7.

20 Fiuza Sanchez F, Silva C, Drumond M, Ferreira E. A formação de valores e a prática da atenção primária na saúde com estudantes de odontologia. Acta bioeth. 2012; 18(1): 101-109.

21 World Health Organization. Continuous improvement of oral health in the 21 st. century the approach of the WHO Global Oral Health Programme. Geneva: WHO; 2003.

22 Spencer AJ, Liu P, Armfield JM, Do LG. Preventive benefit of access to fluoridated water for young adults. J Public Health Dent. 2017; 77(3):263-271.

23 García-Cortés JO, Mejia-Cruz JA, Medina-Cerda E, Orozco G, Medina-Solís CC, Márquez S, Navarrete J, Islas-Granillo H. Experience, prevalence, severity, treatment needs for dental caries and care index in Mexican adolescents and young adults. Rev Invest Clin. 2014; 66(6):505-511.

24 Arrieta K; González F; Díaz S. Historia, severidad de caries y necesidades de tratamiento periodontal en estudiantes de odontología de Cartagena Salud Uninorte 2012; 28(3):364- 
372.

25 Gómez N, Morales M. Determinación de los Índices CPOD e IHOS en estudiantes de la Universidad Veracruzana, México. Rev Chil Salud Pública 2012; 16 (1): 26-31

26 Doncel C, Vidal M, Del Valle M. Relación entre la higiene bucal y la gingivitis en jóvenes. Rev Cub Med Mil. 2011; 40(1): 40-47.

Recibido: 1 de mayo de 2018.

Aceptado: 31 de mayo de 2018. 